\title{
Control of Separation Flow over a Wind Turbine Blade with Plasma Actuators
}

\author{
Erik Potočar ${ }^{1}$ - Branko Širok ${ }^{2}$ - Marko Hočevar² - Matjaž Eberlinc2,* \\ ${ }^{1}$ Ministry of the Economy, Directorate for Energy, Slovenia \\ 2University of Ljubljana, Faculty of Mechanical Engineering, Slovenia
}

This paper analyses the effects produced by a plasma actuator on flow around a turbine blade profile at Re 7600 and Re 10500. The analysis is undertaken with flow visualizations. The experiments indicate that for low Reynolds number plasma has a significant influence on flow around the blade and that the effects of actuation depend on power added to flow and relative distance between the electrode and the separation line of the actuator.

(C)2011 Journal of Mechanical Engineering. All rights reserved.

Keywords: wind turbine blade, plasma actuator, flow control, electrohydrodynamic actuator

\section{INTRODUCTION}

Wind turbines are complex, dynamically flexible structures that must operate under turbulent and unpredictable environmental conditions in which efficiency is highly dependent on welldesigned control. Different significant problems may reduce the aerodynamic performance of blade profiles with low Reynolds number $(R e)$. The possibility to quickly influence the aerodynamic loads acting on individual blades usually operating at low $R e$ depends on active aerodynamic devices. Flow control is focused on the mitigation of these unpredictable environmental conditions by using different strategies like flaps or flexible wings [1] or boundary layer control (e.g. blowing, suction, etc.) [2] and [3]. Inter alia, the concept of reducing blade wake through trailing edge blowing has been investigated in the literature with different explanations being proposed. Schlichting [4] developed a method to prevent flow separation by supplying extra energy to fluid particles.

The use of electrohydrodynamic (EHD) actuators was proposed some years ago [5] and [6]. These actuators ionize air flow and produce localized momentum to the flow through a collision process by migrating charged particles with neutral air particles. EHD phenomena are based on the fact that currents involved are so low that the intensities of the magnetic forces are negligible compared to the electric ones [6]. The main advantages of EHD actuators are absence of moving parts, very short response time and relatively good efficiency in transforming electrical energy to mechanical energy [7]. EHD actuators may be divided into three large groups: corona-based devices [6], dielectric barrier discharge devices [8] and plasma sheet devices [6].

A plasma actuator is composed of two electrodes and a dielectric as shown in Fig. 1. Plasma is generated by dielectric barrier discharge in the area between the exposed electrode and the dielectric when alternating current is applied at high voltage to both electrodes. Plasma is accelerated by the electric field and provides atmosphere with momentum. The device produces a homogeneous luminescence that occupies the inter-electrode space along the span. In this region, the surface is covered by a thin film of ionized air. The flow with several $\mathrm{m} / \mathrm{s}$ of velocity is induced from the exposed electrode to the insulated electrode. If necessary, a third electrode may be added to the system so as to ameliorate the stability of the discharge [9].

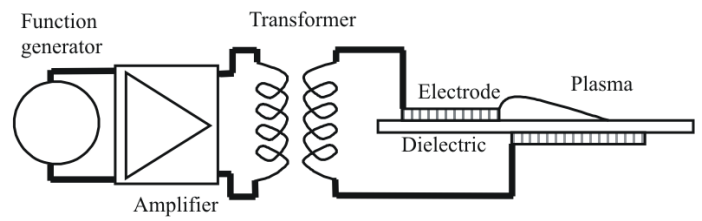

Fig. 1. Measuring equipment

This paper aims at studying the improvement of aerodynamic performance

*Corr. Author's Address: University of Ljubljana, Faculty of Mechanical Engineering, Aškerčeva 6, Ljubljana, Slovenia, matjaz.eberlinc@fs.uni-lj.si 
of wind turbine blade operating at low Re by means of a plasma sheet device powered by the AC potential difference. The purpose of measurement performed on a wind turbine blade in low-speed wind tunnel is to confirm the influence of plasma actuator on the flow field near the upper part of the profile. The purpose is to better understand changes occurring to the aerodynamic characteristic under plasma actuators and to develop wind turbine blades with better aerodynamic characteristic.

The influence of plasma actuators on flow around the blade depends on flow conditions (power of plasma, flow velocities, angles of attack, etc.). A good synthesis [5] of influence of plasma actuators can be obtained by analyzing the intensity level of actuation as a function of angle of attack of turbine blade profile $\alpha$ and velocity $U_{0}$. The intensity of actuation is evaluated with non-dimensional power coefficient $C_{W}$ defined as follows [9]:

$$
C_{W}=\frac{W}{0.5 \rho U_{0}^{3} b \delta}\left(\frac{L_{\text {ele }}}{c}\right)^{2},
$$

where $L_{\text {ele }}$ is inter-electrode distance, $b$ electrode length, $\delta$ thickness of plasma sheet and $c$ blade profile cord. Parameter $W$ is the power of discharge and may be easily calculated as the product of discharge current $I$ and the applied voltage difference between electrodes $\Delta V$.

The same power coefficient in terms of other usual non-dimensional parameter can be expressed as follows:

$$
C_{W}=\frac{E_{H D}}{\operatorname{Re}^{2}} \frac{v_{i}}{U_{0}}\left(\frac{L_{e l e}}{c}\right)^{2},
$$

where $\operatorname{Re}=\left(U_{0} \cdot C\right) / v, E_{H D}=\left(I c^{3} \mu_{i}\right) /\left(A \rho \vartheta^{2}\right)$ and $A=b \cdot \delta$ are the discharge section, $\rho$ and $v$ respectively the density and kinematic viscosity of the fluid, and $\mu_{i}$ the mobility of ions. Ion velocity $v_{i}$ in Eq. (2) can be estimated with $\mu_{i} E_{0} \approx \mu_{i}\left(\Delta V / L_{\text {ele }}\right)[10]$.

\section{EXPERIMENTAL SET-UP}

Experimental set-up is shown in Fig. 2. It is composed of a wind tunnel, a blade profile, and plasma actuation device and flow visualization equipment.

\subsection{Wind Tunnel and Blade Profile}

The experiments were conducted in an open wind tunnel consisting of a square closed test section, as shown in Fig. 2. Air is drawn into the tunnel through the converging section with area ratio $16: 1$ and with inlet size of $400 \times 400$ $\mathrm{mm}$ (Fig. 2, point 2). The converging section included settling screens. A straight square test section $800 \times 100 \times 100 \mathrm{~mm}$ in size (Fig. 2, point 4) was used, which accommodated the profile. A honeycomb section formed the end of the test section of the wind tunnel (Fig. 2, point 10) to provide the stationary vortex-free flow field in the test section area. The test section was connected to the inlet of the centrifugal fan by a flexible pipe. A HFR 140-17D type radial fan was applied (Fig. 2, point 8 ) and was controlled by a frequency inverter regulator (Fig. 2, point 9).

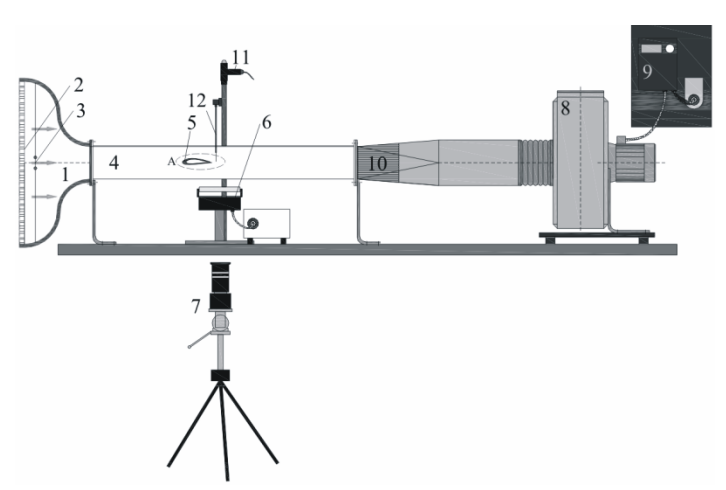

Fig. 2. Wind tunnel experimental set-up of the test section [15]; 1. converging section $400 \times 400$ $\mathrm{mm}$, 2. settling screens at the inlet of wind tunnel,

3. smoke generator, 4. test section-perspex window channel $800 \times 100 \times 100 \mathrm{~mm}$, 5. blade

NACA 4421, 6. illumination, 7. high-speed camera, 8. centrifugal fan, 9. frequency regulator, 10. honeycomb, 11. positioning table, 12. second illumination

Volume flow rate in the wind tunnel can be set between 0 and $650 \mathrm{~m}^{3} / \mathrm{h}$. In addition, with a 2-component hot-wire anemometer, velocity field in the test section area was studied [11]. This allowed for determination of unperturbed velocity field in the test section area of the wind tunnel. The measurements showed uniform velocity profiles through the channel of the test section 
area with mean turbulence intensity being less than $1.5 \%$ [11].

A NACA 4421 profile with $30 \mathrm{~mm}$ chord length and aspect ratio of 3.03 was used to profile the wind turbine blade. The profile was made out of ABS plastic, printed with 3D printer. The blade profile was located in the middle of the test section area (Fig. 2, point 5) and was supported by both vertical walls of the test section. Profile NACA 4421 has been chosen in order to better demonstrate the impact of plasma to a single profile of the wind turbine [11].

\subsection{Plasma Actuating Device}

A plasma actuating device consists of two high voltage and high frequency wires, which enter the hollow profile through a hole on one side of the chord. On the surface of the upper part of profile, four EHD actuators as shown in Fig. 3 are placed.

The tested EHD plasma actuators (power supply/actuator schematic is shown in Fig. 3) consist of conductive copper strips separated by a 50 micron thick Kapton dielectric (dielectric strength $118 \mathrm{kV} / \mathrm{mm}$ ). Typical widths of $5 \mathrm{~mm}$ with corresponding lengths of $70 \mathrm{~mm}$ were used. A function generator is used to provide sinusoidal input to amplifier power supply. The output drives an inductively matched transformer, capable of $6 \mathrm{kV}$ output with 1 to $250 \mathrm{~V}$ RMS input at 4 to $11 \mathrm{kHz}$. The input power was monitored by oscilloscope.

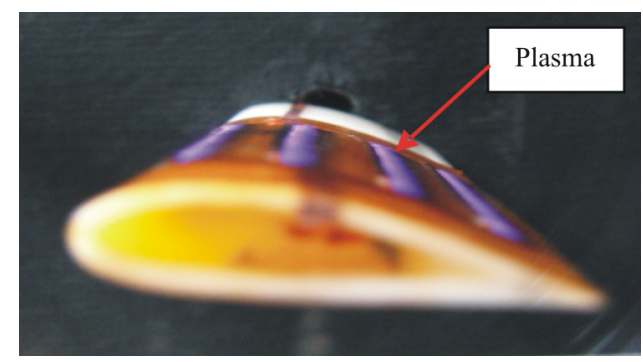

Fig. 3. Plasma on wind turbine profile

\subsection{Flow Visualization}

Measurements with flow visualization were performed by introducing passive tracer smoke into a fully developed flow. To visualize the flow, a passive tracer smoke consisting of vaporized paraffin oil was used. The smoke is made with thin resistance wire of $0.1 \mathrm{~mm}$, which was heated. For generation of the passive tracer smoke we used paraffin oil drops on a wire. The wire was mounted on the walls of the converging section. A presumption was made that passive tracer smoke particles ideally follow the flow and that image intensity is proportional to the concentration of the passive tracer smoke [10]. The passive tracer smoke flow was evaluated with appropriate equipment for calculating intensity profiles [11]. Camera Dragonfly Express IEEE1394b with Edmund Optics 75 mm Double Gauss lens was used for image acquisition, with image acquisition frequency of $400 \mathrm{~Hz}$. Recorded images had 8-bit grey level depth, resolution of $613 \times 200$ pixels, and were captured with shutter speed of $0.5 \mathrm{~ms}$. The camera was placed perpendicularly to the profile surface at the distance of $2 \mathrm{~m}$ from the test section of the wind tunnel [12] and [13].

For illumination, two Vega Velum DC 150 W (Fig. 2, point 6) continuous flicker-free light sources with line light guide were used. Software package LabView with Vision toolkit was used for setting up the camera, acquisition and storage of digital images. For each measurement point, 800 successive images were taken. The number of images was limited by the time of uniform passive tracer smoke generation. Overall, uncertainty of measurements of instantaneous passive tracer concentration was estimated at $3.8 \%$ of the measured value [11].

\subsection{Methodology Behind Analyzed Image Sequences}

Computer-aided visualization allows time and space measurements of flow structures to study flow around the blade profile in the wind tunnel. Algorithms for image analysis are discussed below [10] and [14]. The Software package enabled the transformation of the snapshot format from AVI to BMP. Image sequences were then analyzed by Dynascan software. A study window in the program was placed above each image. The window was divided into a matrix $i \times j=40 \times 22$ of equal cells. Each cell had a rectangular form $d x_{c} \times d y_{c}=10 \times 10$ pixels in size. For every image in the sequence, average grey level $\left(A_{c}\right)$ in each 
window cell was calculated using the following equation [15]:

$$
A_{c}(i, j, n)=\frac{1}{d y_{c} d x_{c}} \sum_{i_{p}=1}^{d y_{c}} \sum_{j_{p}=1}^{d x_{c}} E_{p}\left(i+i_{p}, j+j_{p}, n\right),
$$

where $E_{p}\left(i+i_{p}, j+j_{p}\right)$ represents the grey intensity of the observed pixel at cell position $(i, j)$ and relative position $\left(i_{p}, j_{p}\right)$ of the pixel within cell location; $n$ denotes the consecutive number of image in the sequence. The intensity of pixels $E_{p}\left(i+i_{p}, j+j_{p}\right)$ ranges from 0 (black) to 255 (white). The mean value of the grey intensity in each cell for the whole sequence of images is obtained by the following formula:

$$
\mu_{c}(i, j)=\frac{1}{N} \sum_{n=1}^{N} A_{c}(i, j, n)
$$

where $N$ denotes the number of all images in the sequence; there were 800 images in each sequence. The results demonstrate a transition in grey levels from a low to a high value on the boundary layer of the profile.

\section{EXPERIMENTAL RESULTS}

\subsection{Flow Visualization}

The experiments were made for two working points, presented in Table 1 below, which show the effect of discharge on aerodynamic properties at different flow conditions.

Table 1. Aerodynamic non-dimensional coefficient as a function of flow conditions and actuation intensity

\begin{tabular}{|c|c|c|c|}
\hline $\begin{array}{c}\text { Flow } \\
\text { condition }\end{array}$ & $\alpha\left(^{\circ}\right)$ & $R e$ & $\begin{array}{c}C_{w}\left(\times 10^{3}\right) \\
\text { max. voltage } 6 \mathrm{kV}\end{array}$ \\
\hline 1 & 7 & 7600 & 22.5 \\
\hline 2 & 7 & 10500 & 11.9 \\
\hline
\end{tabular}

The angle of attack of the turbine blade profile is set at $7^{\circ}$. This angle of attack influences the flow most visibly. For the first working point, stream velocity was $4 \mathrm{~m} / \mathrm{s}$; for the second working point it was $5.5 \mathrm{~m} / \mathrm{s}$. For each working point, flow visualization was captured in several measuring points. Corresponding Re based on free stream velocity and the chord lengths are $\operatorname{Re} 7600$ and
Re 10500. The effects of discharge on the blade profile depend on characteristics of the considered regime.

For each working point, flow visualization was captured in several measuring points: (i) without plasma actuators and (ii) with plasma actuators. For measuring points with plasma actuators, different intensities of voltage on actuator and voltage on amplifier were used, as presented in Fig. 4.

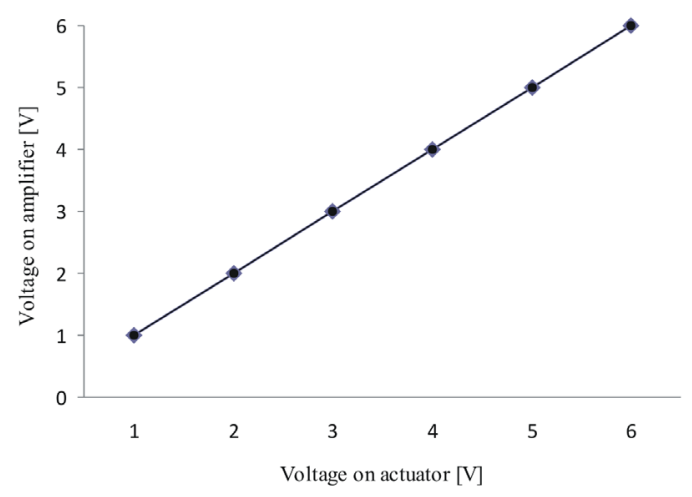

Fig. 4. Correlations between voltage on actuator and voltage on amplifier

Fig. 4 shows the present input conditions. It should be noted that input energy increases as frequency or voltage increases.

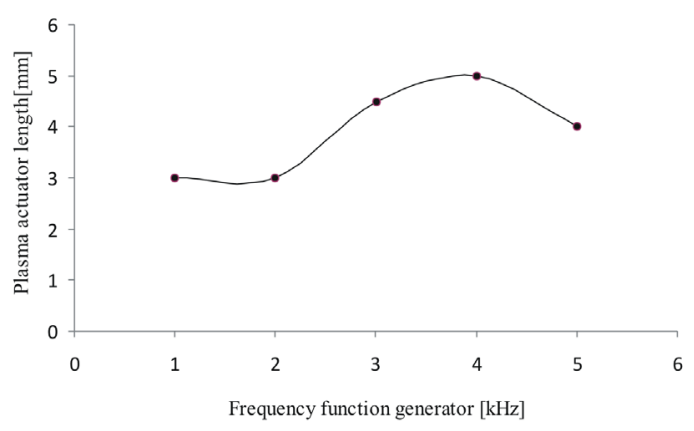

Fig. 5. Correlations between frequency and plasma width on actuator

The size of plasma on the blade profile was analyzed, based on digital images obtained during measurements of flow visualization.

The results presented in Fig. 5 show that plasma has the highest rate and influence on the flow at frequency value of $3.5 \mathrm{kHz}$. 
Different measuring points, in which flow visualization measurements were performed, are presented in Table 2. The range of velocities and frequencies tested in our sets of experiments are presented by four examples: (1) Flow over the blade fully attached to the surface with plasma, (2) Flow over the blade in dependence of different $R e$ and plasma intensity, (3) Flow over the blade with first plasma line and (4) Behavior of the passive tracer smoke near plasma.

Table 2. Conditions during the cases

\begin{tabular}{|c|c|c|}
\hline $\begin{array}{c}\text { Measurement } \\
\text { cases }\end{array}$ & $\operatorname{Re}$ & $\begin{array}{c}\text { Frequency Function } \\
\text { Generator }[\mathrm{kHz}]\end{array}$ \\
\hline 1 & 7600 & 4 \\
\hline 2 & 7600 & 5 \\
\hline 3 & 7600 & 6 \\
\hline 4 & 7600 & without \\
\hline 5 & 10500 & 4 \\
\hline 6 & 10500 & 5 \\
\hline 7 & 10500 & 6 \\
\hline 8 & 10500 & without \\
\hline
\end{tabular}

2.1.1 Flow over the Blade Fully Attached to the Surface with Plasma

In the low range of $R e<3 \times 10^{4}$, and using NACA 4421 blade profile, the flow conditions normally do not include laminar boundary layer towards the profile trailing edge [11]. When the blade profile is inclined at the angle of attack of $7^{\circ}$, separation position is expected to occur in the middle of the blade profile [16] and [17]. Flow visualization results without the plasma actuator are shown in Fig. 6. The separation of flow on the suction side of the blade occurs (Fig. 6, point A). External flow is not able to follow the path of the blades' profile; therefore, it separates from the profile. It is expected that with plasma actuators (Fig. 7), the flow will better follow the shape of the blade near the trailing edge. Qualitative changes of flow structures over the blade appear in both cases. In the case of flow field in Fig. 7, no vortex is observed. This is due to the presence of the flow of plasma that acts as an ejector. It locally reduces pressure near the trailing edge due to higher velocity. The adverse pressure gradient is thus reduced, the boundary layer cannot thicken and no flow separation occurs. Consequently, plasma contributes to preservation of flow direction and stabilization of flow structures. The flow is forced to follow the path of the blade.

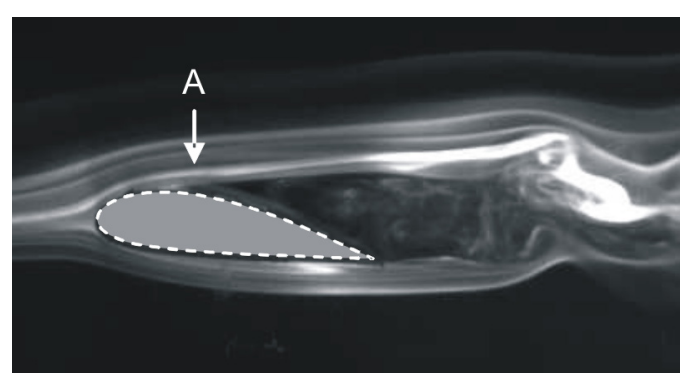

Fig. 6. Flow visualization (Case 8)

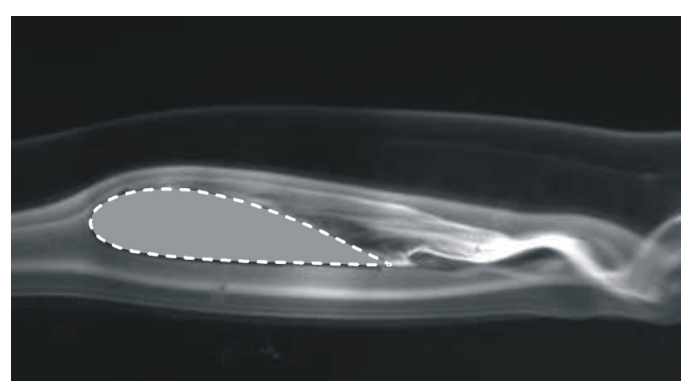

Fig. 7. Flow visualization (Case 6)

\subsubsection{Flow over the Blade in Dependence on Different Re and Plasma Intensity}

Figs. 8 to 16 present flow visualization of partially separated flow configuration obtained at $\alpha=7^{\circ}$ at $\operatorname{Re} 7600$ and $\operatorname{Re} 10500$. Flow separation without plasma (Fig. 8) was revealed by the passive tracer smoke where the separation point lies at $x / c \approx 0.3(x$ - distance from the front part of the blade to the separation point, $c$ - chord length of the blade). Again, similar observation can be made when compared to results in Figs. 6 and 7. Based on Fig. 8, the location of the separation point and the passive tracer smoke can be estimated. From the point of separation (Fig. 8, point $\mathrm{B}$ ), the distance between the passive tracer smoke and the blade profile surface continuously increases.

When plasma was applied in Case 1 (Fig. 9), the angle of the passive tracer smoke path was significantly smaller and oriented in the direction of the blade's trailing edge. Therefore, the flow direction is changed and the flow field around the 
blade in the presence of plasma follows better the profile. Plasma decreased the circulating flow and vortex formation on the suction side of the blade wall.

In Case 2 (Fig. 10) with the highest rate of plasma, the passive tracer smoke follows the path of the blade profile and the separation of the flow decreases.

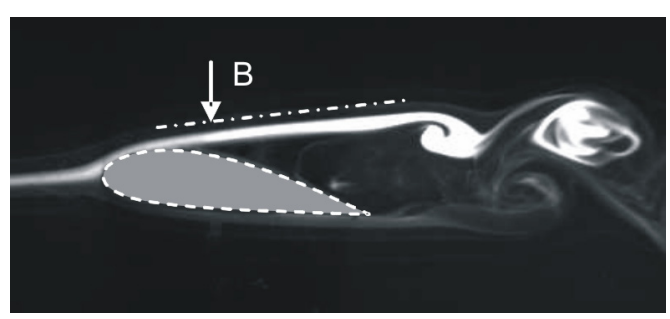

Fig. 8. Flow visualization (Case 4)

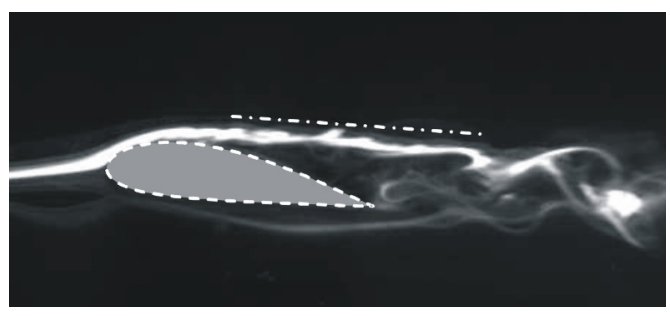

Fig. 9. Flow visualization (Case 1)

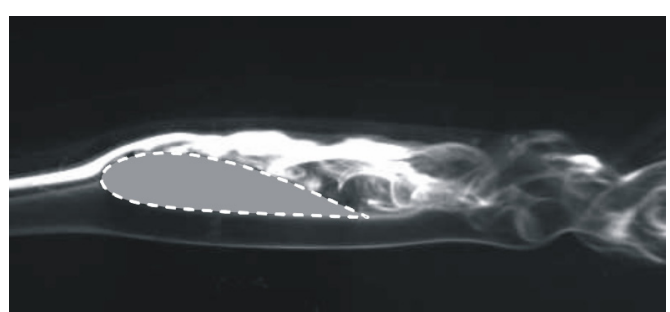

Fig. 10. Flow visualization (Case 2)

Digital image sequences of the passive tracer smoke for cases with and without plasma actuator at two different velocities were then analyzed with Dynascan software. A window $(40 \times 22)$ of equal cells was placed above each image. For every image in the sequence, average grey level $\left(A_{c}\right)$ in each window cell was calculated in accordance with Eq. (3). The mean value of grey intensity in each cell for the whole sequence of images was obtained from Eq. (4).

Results presented in Figs. 11 to 16 show plasma's influence on the passing of the flow through the blade profile. Plasma has the strongest influence in Case 2 (Fig. 12) because the passive tracer smoke presenting the flow around the blade profile is fully reattached. In cases without plasma (Figs. 13 and 16), conclusions are similar. They confirm the results from the quality assessment of the digital images. As expected, plasma contributes to the preservation of the direction of the flow and stabilization of flow structures. The flow is forced to follow the path of the blade; hence, mass flow near the trailing edge increases. This phenomenon leads to conclude that the flow direction change and flow field around the blade in the presence of plasma better follows the profile. As a result, the following estimation can be made: plasma used in this case has a positive influence; it prevents increases in pressure difference, decreases formation of circulating flow and vortex on the suction side of the blade wall. All this can also be reflected in decreased energy dissipation.

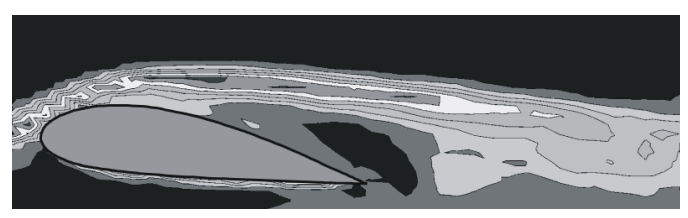

Fig. 11. Flow visualization (Case 1)

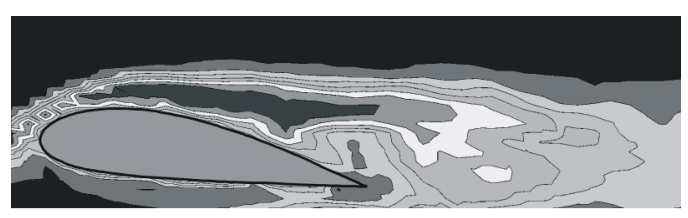

Fig. 12. Flow visualization (Case 2)

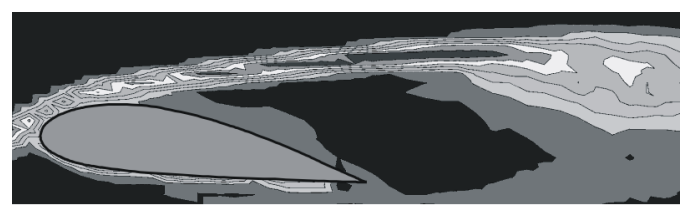

Fig. 13. Flow visualization (Case 4)

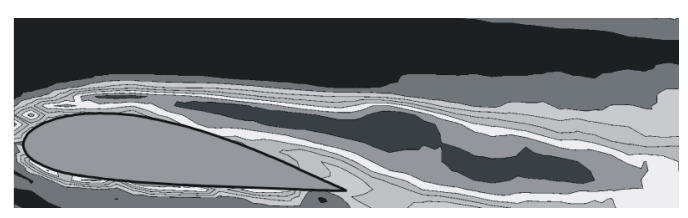

Fig. 14. Flow visualization (Case 6) 


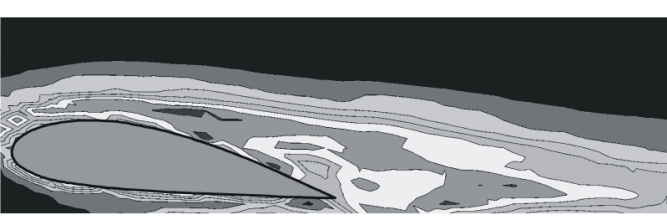

Fig. 15. Flow visualization (Case 7)

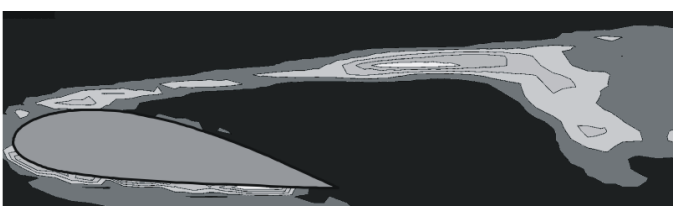

Fig. 16. Flow visualization (Case 8)

\subsubsection{Flow over the Blade with First Plasma Line}

A set of flow conditions at $\operatorname{Re} 7600$, that leads to separation of the boundary layer close to the leading edge was analyzed (Fig. 17, Case 4 vortex separation without plasma and Fig. 18, Case 2 with plasma).

Fig. 17 presents a continuous increase in distance of the passive tracer smoke from the blade profile, which represents the boundary layer separation. Similar estimation can be made as in previous cases: the separation point occurs at $x / c \approx 0.2$. When the flow separates from the blade profile, the separated shear layer rapidly undergoes a transition to turbulent flow. Vortex position and size depend on characteristics of the blade, $R e$, the angle of attack and frequency of plasma.

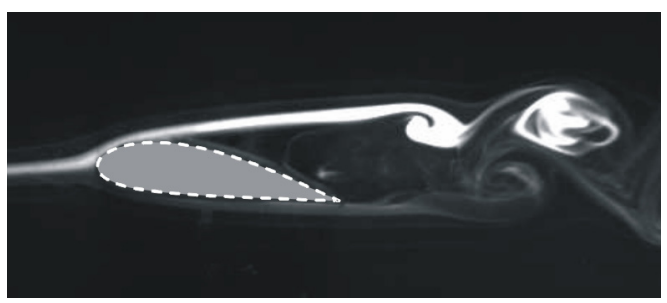

Fig. 17. Flow visualization (Case 4)

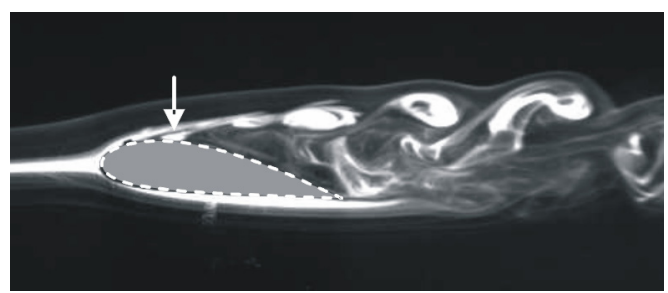

Fig. 18. Flow visualization (Case 2)
In Fig. 18 close to the separation point where plasma actuator is attached, quality differences occur between cases with and without plasma. When using the maximum power of plasma, but only with one line of plasma as in Case 2 in Fig. 18, the frequency of vortex generation changes. The position of flow separation from the blade profile seems to be unchanged and the flow from trailing edge to the leading edge remains present when comparing cases with and without plasma. Nevertheless, it seems that only a small amount of plasma applied yields a noticeable difference. The efficiency of plasma is therefore increased when applied on the entire section of the blade profile.

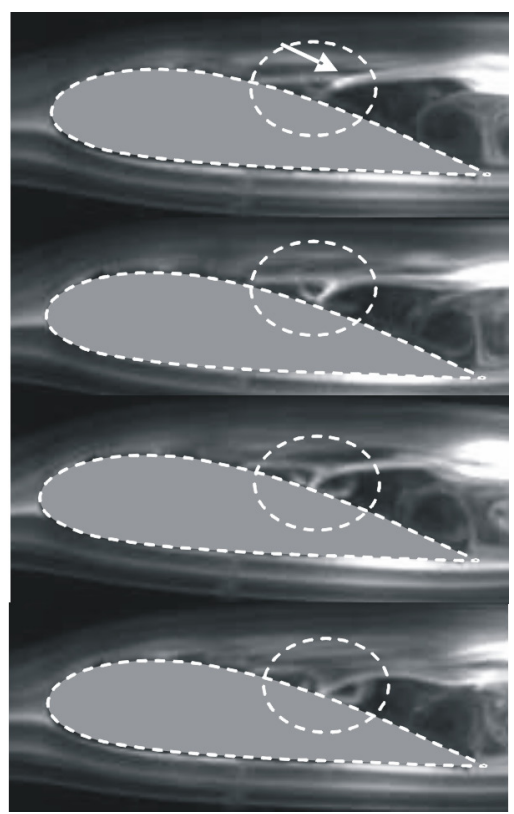

Fig. 19. Flow visualization (Case 5)

\subsubsection{Behavior of passive Tracer Smoke near Plasma}

A sequence of digital images with plasma in Fig. 19 reveals changes around the individual plasma actuator. In the first digital image, the direction of the passive tracer smoke (marked with arrow) is redirected closer to the profile surface due to the influence of plasma. The third digital image in the sequence reveals the formation of small vortex in the direction of the blade profile. 
This is a result of plasma influencing the flow around the blade.

The strong electric field ionizes air molecules in the region above the insulated electrode, forming plasma which extends from the exposed electrode along the entire insulated electrode. Plasma induces velocity in the surrounding fluid. The electron movement ionizes the air, generating charged particles which are subsequently accelerated through the electric field. The momentum of the accelerated charged particles is then transferred to the surrounding air through collisions with neutral particles. This momentum transfer occurs very close to the surface of the actuator itself, typically in the subboundary layer region [7]. Based on these results, it was considered that the actuator produces enough strength to partly or fully reattach the flow.

\section{CONCLUSIONS}

Within the present experimental circumstances, the following facts can be observed:

- input energy (correlation of frequency and voltage,) calculated as the product of discharge current $I$ and applied voltage difference between electrodes $\Delta V$, has a strong influence on the flow around the turbine blade.

- Plasma actuators can control the separation point of the flow, passing the blade profile.

- Input energy (max. $20 \mathrm{~W}$ in length of $10 \mathrm{~cm}$ of plasma) represents a sufficient amount of energy to control the flow over the blade in the applied/established conditions.

More extensive research will be carried out in the future. We will focus on quantity assessment of the blade flow field. Generating plasma on a small scale laboratory wind turbine will enable us to measure the differences in aerodynamic and acoustic characteristics between cases with and without plasma. We believe that plasma control on a wind turbine will have similar influence as presented in this paper. However, some changes may be considered due to additional forces acting on the flow around the rotating blade.

\section{REFERENCES}

[1] Shyy, W., Berg, M., Ljungqvist, D. (1999). Flapping and flexible wings for biological and micro air vehicles. Progress in Aerospace Sciences, vol. 35, no. 5, p. 455-505, DOI:10.1016/S0376-0421(98)00016-5

[2] Zaman, K., McKinzie, D.J. (1991). Control of laminar separation over blade profiles by acoustic excitation. American Institute of Aeronautics and Astronautics Journal, no. 7 , p. 1075-1083.

[3] Greenblatt, D., Wygnanski, I. (2000). Use of periodic excitation to enhance blade profile performance at low Reynolds numbers. Journal of Aircraft, vol. 1, p. 190-192.

[4] Schlichting, H., (1979). Boundary-Layer Theory, Springer, Berlin.

[5] D'Adamo, J., Artana, G., Moreau, E., Touchard, G. (2002). Control of the airflow close to a flat plate with electrohydrodynamic actuators. ASME Conference Proceedings, vol. 1, p. 1339-1344.

[6] Colver, G., El-Khabiry, S. (1999). Modeling of DC corona discharge along an electrically conductive flat plate with gas flow. IEEE Transactions on Industry Applications, vol. 35, p. 387-394, DOI: 10.1109/28.753633.

[7] Roth, J.R., Sherman, D.M. (2000). Electrohydrodynamic flow control with a glow discharge surface plasma. American Institute of Aeronautics and Astronautics Journal, vol. 38, no. 7, p. 1166-1178.

[8] Louste, C., Artana, G., Moreau, E., Touchard, G. (2005). Sliding discharge in air at atmospheric pressure: electrical properties. Journal of Electrostatics, vol. 63, p. 615-620, DOI: 10.1016/j.elstat.2005.03.026.

[9] IEEE-DEIS-EHD Technical Committee (2003), Dielectrics and Electrical Insulation, IEEE Transactions, vol. 10, p. 3-6.

[10]Eberlinc, M., Dular, M., Širok, B., Lapajne, B. (2008). Influence of blade deformation on integral characteristic of axial flow fan. Strojniški vestnik - Journal of Mechanical Engineering, vol. 54, no. 3, p. 159-169.

[11] Eberlinc, M., Širok, B., Hočevar, M. (2009). Experimental investigation of the interaction of two flows on the axial fan hollow blades by flow visualization and hot-wire anemometry. 
Experimental Thermal and Fluid Sciences, vol. 33, p. 929-937, DOI:0.1016/j. expthermflusci.2009.03.012.

[12] Benedik, G., Širok, B., Rihtaršič, J., Hočevar, M. (2010). Flow characteristics of bladeless impeller made of open cell porous material. Strojniški vestnik - Journal of Mechanical Engineering, vol. 56, no. 7/8, p. 464-476.

[13] Chen, J., Hočevar, M., Širok, B. (2011) Melt volume flow measurement in the mineralwool production process. Strojniški vestnik - Journal of Mechanical Engineering, vol. 57, no. 4, p. 293-303, DOI:10.5545/svjme.2010.159.

[14] Osterman, A., Hočevar, M., Širok, B., Dular, M. (2009). Characterization of incipient cavitation in axial valve by hydrophone and visualization. Experimental Thermal and Fluid Science, vol. 33, no. 5, p. 929-937.

[15] Širok, B., Bajcar, T., Dular, M. (2002). Reverse flow phenomenon in a rotating diffuser. Journal of Flow Visualization \& Image Processing, vol. 9, p. 1-18.

[16] Benard, N., Braud, P., Touchard, G., Moreau, E. (2008). Detachment and attachment of an axisymmetric non-reactive jet with turbulent shear layer: control by plasma actuator. Experimental Thermal and Fluid Science, vol. 32, no. 6, p. 1193-1203, DOI: $0.1016 /$ j. expthermflusci.2008.01.010.

[17] Lemire, S., Vo, H.D., Benner, M.W. (2009). Performance improvement of axial compressors and fans with plasma actuation. International Journal of Rotating Machinery, p. 1155. 